\title{
Association Mapping and Domestication Analysis to Dissect Genetic Improvement Process of Upland Cotton Yield-Related Traits in China
}

\section{GUO Chunping}

Shihezi University College of Agriculture

\section{PAN Zhenyuan}

Shihezi University College of Agriculture

YOU Chunyuan

Shihezi University College of Agriculture

\section{ZHOU Xiaofeng}

Xinjiang Academy of Agricultural and Reclamation Science

\section{HUANG Cong}

Huazhong Agriculture University

\section{SHEN Chao}

Huazhong Agricultural University: Huazhong Agriculture University

\section{ZHAO Ruihai}

Shihezi University College of Agriculture

\section{YANG Qingyong}

Shihezi University College of Agriculture

ZHU Longfu

Shihezi University College of Agriculture

\section{LIN Zhongxu}

Shihezi University College of Agriculture

Xinhui Nie ( $\square$ xjnxh2004130@126.com)

Shihezi University College of Agriculture

\section{Research}

Keywords: upland cotton, genome wide association study, yield related traits, favorite alleles

Posted Date: February 15th, 2021

DOl: https://doi.org/10.21203/rs.3.rs-196227/v1 
License: (c) (i) This work is licensed under a Creative Commons Attribution 4.0 International License. Read Full License

Version of Record: A version of this preprint was published at Journal of Cotton Research on May 4th, 2021. See the published version at https://doi.org/10.1186/s42397-021-00087-3. 


\section{Abstract}

\section{Background}

Cotton fiber yield is a complex trait, which can be influenced by multiple agronomic traits. Unravelling the genetic basis of cotton fiber yield related traits contributes to genetic improvement of cotton fiber.

\section{Results}

In this study, 503 upland cotton varieties covering the 4 breeding stages in China were used to for association mapping and domestication analysis of upland cotton yield-related traits in China. 140 quantitative trait loci (QTLs) significantly associated with ten fiber yield related traits were identified, among which, 29 QTLs showed an increasing trend contribution to cotton yield-related traits from BS1 to BS4, and 26 QTLs showed decreased trend effect. 4 favorable alleles of 9 major QTLs $\left(R^{2} \geq 3\right)$ were strongly selected during the breeding stages, and the candidate genes of the 4 strongly selected alleles were predicated according to the gene function annotation and tissue expression data.

\section{Conclusions}

The study not only uncovers the genetic basis of 10 cotton yield related traits, but also provides genetic evidence for cotton improvement during the cotton breeding process in China.

\section{Background}

Cotton is one of the most important industrial crop in the word, which has been cultivated for over 7000 years, providing an important raw material for the textile industry (Fang et al. 2017a; Maik et al. 2015). Among the 4 cultivated cotton species, Gossypium hirsutum (upland cotton) is the most widespread species due to the high adaptability and yield, which takes up approximately $95 \%$ of cotton production in the word (Chen et al. 2007). Hence, the genetic improvement of upland cotton to increase cotton fiber yield is one of the important goals of cotton breeding.

Fiber yield is a complex trait, which can be influenced by multiple traits, including seed cotton weight (SCW), lint weight (LW), lint percentage (LP), effective boll number (EBN), plant height (PH), first fruit spur height (FFSH), fruit spur branch number (FSBN), first fruit branch position (FFBP), flowering period (FP), and whole growth period (WGP) (Li et al. 2018b; Sun et al. 2018). Unravelling the genetic basis of cotton fiber yield related traits contributes to cotton fiber production increases. Based on linkage analysis, many QTL for cotton yield traits have been identified (An et al. 2010; Gore et al. 2014; Liu et al. 2012). However, the construction of linkage population used for fine mapping is a time-consuming process, such as recombinant Inbred Lines (RIL), and near-isogenic line (NIL) (Li et al. 2018a; Zhang et al. 2020). In recent years, genome-wide association studies (GWAS), which is based on linkage disequilibrium (LD), has been widely used in plants to identify various traits related QTLs by using the natural populations (Huang et al. 2016; Mengistu et al. 2016; Yang et al. 2014). GWAS has also been conducted for the genetic dissection 
of cotton yield related traits in cotton. 251 significant loci were detected to be associated with lint yield yields by using 651 simple sequence repeats (SSRs) and 323 accessions of Gossypium hirsutum L (Jia et al. 2014). 13 cotton yield related QTL and 44 cotton fiber quality related QTL were detected respectively by using 198 SSR markers and 302 elite Upland cotton accessions (Ademe et al. 2017). 43 marker loci were detected to be associated with cotton yield traits by using 201 pleomorphic markers and 403 accessions (Dong et al. 2018). The identification of cotton yield related QTL lays the foundation for gene cloning and marker assisted selective (MAS) breeding.

The natural population resources possess widely genetic variation, which are also suitable to study species domestication, such as maize (Hufford et al. 2012; Xue et al. 2016), rice (Zheng et al. 2019), soybean (Zhang et al. 2019; Zhou et al. 2015), tomato (Soltis et al. 2019), watermelon (Zhao et al. 2019), and cotton (Du and Huang, 2018; Nie et al. 2020). During crop breeding process, the favorable alleles were constantly enriched, so the varieties collected from different breeding stages could help us uncover the artificially selected loci. In this study, 503 upland cotton varieties covering the 4 breeding stages in China were used to construct a natural population. 10 cotton yield related traits were investigated in multiple environments. An association analysis was performed based on best linear unbiased predictions (BLUP) data from multiple environments, and 176 polymorphic SSRs, after which, the identified QTL were used for domestication analysis and favorable alleles selection. 3 major QTL for LP and one QTL for FS and WGP were strongly selected during the four breeding stages. This study aimed to explore the genetic architecture of 10 cotton yield related traits, and to uncover the genetic improvement during the cotton breeding process in China.

\section{Materials And Methods}

\section{Materials}

In this study, 503 upland cotton inbred cultivars cultivated in, or introduced to China were collected to construct the natural mapping population (Table S1), which represent extensive genetic variation resources related to fiber yield related traits and covered four breeding stages in China.

\section{Field experiments and phenotype data collection}

Ten cotton yield-related traits of the 503 cotton inbred cultivars were measured under multiple environments. 5 phenotypic data, including SCW, LW, LP, EBN, and PH were collected from 4 areas of China (Shihezi (SHZ), Xinjiang; Kuerle (KEL) Xinjiang; Yuanyang (YY), Henan; Huanggang (HG), Hubei) in 2012 and 2013. The phenotypic data of FFSH, and FSBN were collected from 4 areas of China (SHZ, KEL and YY) in 2012 and 2013. The phenotypic data of FFBP, FP, and WGP, were collected from 2 areas of China (SHZ and YY) in 2012 and 2013. The experiment followed a randomized complete block design with single row plot and two replications.

\section{Phenotypic data analysis}


The variance, correlation, and repeatability analysis of the phenotypic data in multiple environment were conducted by $\mathrm{R}$ programming language.

Best linear unbiased predictions (BLUP) were used to estimate phenotypic traits across multiple environments based on a linear model, which were conducted by R software (http://www.r-project.org).

The basic statistical analysis of phenotypic data, including minimum (Min), maximum (Max), mean, standard deviation (SD), and coefficient of variation (CV), were conducted by IBM SPSS Statistics 21.

\section{Association analysis}

179 polymorphic SSR markers covering the whole genome were taken from the previous study, where the linkage disequilibrium and population structure analysis were also done (Nie et al., 2016). TASSEL V2.1 software was used to detect the association relationship between BLUP phenotypic data and genotypic data in three models, including GLM $(P+G)+Q, G L M(P+G)+P C A$ and $M L M(G+P+Q+K)$. The $P$ values of markers associated with QTL were regulated by the method of multiple testing correction by controlling the false discovery rate (Benjamini and Hochberg, 1995).

\section{Effect and evolution analysis of the QTLs in four breeding stages, and analysis favorable alleles for major QTL}

The effect of the identified QTL were evaluated in the four breeding stages based on the ten traits. 9 major QTLs $\left(R^{2} \geq 3\right)$ were used for accumulation of favorable alleles analysis. The effects of different alleles were evaluated by using the phenotypic data, after that, the favorable alleles were used for frequency analysis in the four breeding stages, as well as the favorable alleles carrier materials selection.

\section{Candidate gene annotation and prediction}

The 4 strongly selected alleles associated to cotton yield-related traits were mapped to the TM- 1 genome. The candidate regions for marker-trait loci were set around the LD decay distance as $400 \mathrm{~kb}$. The genes in the candidate regions were used for key candidate genes selection by using annotation.

\section{Gene expression patterns}

The tissue expression levels of the candidate genes were obtained from previously reported transcriptome data (Zhang et al. 2015).

\section{Results}

\section{Performances of cotton yield-related traits of the $\mathbf{5 0 3}$ upland cotton germplasm resources in multiple environments}

BLUP was used to determine the phenotypic data of cotton yield-related traits in multiple environments (Table S2), which was used for association analysis. The average phenotypic coefficient of variation (CV) 
for 10 yield traits ranged from $2.85 \%$ (WGP) to $12.31 \%$ (FFSH) (Table 1). The highest CV was observed in FFSH (12.09\%), the lowest in WGP (2.28\%). The highest heritability was in LP (0.93), and the lowest in EBN and FSBN (0.60 and 0.46$)$, ranging 0.75 to 0.87 in the other seven traits (Table 1 ).

Table 1 Statistics of cotton yield-related traits in the 503 upland cotton germplasm resources

\begin{tabular}{|lllllll|}
\hline & Min & Max & Mean & SD & CV\% & H2 \\
\hline SCW (g) & 3.73 & 5.87 & 4.87 & 0.3 & 6.18 & 0.81 \\
\hline LW (g) & 1.19 & 2.4 & 1.88 & 0.19 & 9.89 & 0.87 \\
\hline LP (\%) & 27.07 & 47.38 & 38.31 & 3.04 & 7.92 & 0.93 \\
\hline EBN (bolls) & 13.33 & 21.74 & 17.44 & 1.37 & 7.87 & 0.6 \\
\hline PH (cm) & 72.44 & 116.17 & 92.81 & 5.54 & 5.97 & 0.76 \\
FFSH (cm) & 8.67 & 21.34 & 13.75 & 1.69 & 12.31 & 0.75 \\
\hline FSBN (branches) & 8.24 & 10.23 & 9.31 & 0.27 & 2.9 & 0.46 \\
\hline FFBP (nodes) & 4.43 & 6.92 & 5.85 & 0.41 & 6.97 & 0.75 \\
\hline FP (days) & 56.26 & 68.17 & 62.68 & 2.56 & 4.09 & 0.84 \\
\hline WGB (days) & 109.09 & 126.28 & 117.99 & 3.36 & 2.85 & 0.79 \\
\hline
\end{tabular}

The phenotypic trends of yield-related traits are shown in Fig. 1. Among them, LP (Fig.1c) showed most stable in the 8 environment; SCW (Fig. 1a), LW (Fig. 1b), EBN (Fig. 1d), PH (Fig. 1e), FFSH (Fig. 1f), FSBN (Fig. 1g), FFBP (Fig. 1h), and WGP (Fig. 1j) were relatively stable in the same site between different years; SCW (Fig. 1a) and LW (Fig. 1b) in HG showed relatively lower than other sites, while EBN (Fig. 1d) and PH (Fig. 1e) showed relatively higher in HG; FFSH (Fig. 1f) in HN showed relatively lower than other sites, while FSBN (Fig. 1g) showed relatively higher in HN.

The correlations between two environments were obtained among multiple environments for the ten cotton yield-related traits (Fig. 2). Among the 10 yield-related traits, the average correlations between environments were ranked as LP (0.62)> FP (0.57)> WGP (0.51) > FFBP $(0.45)>\operatorname{LW}(0.43)>\mathrm{FFSH}(0.38)>$ SCW (0.32)> PH (0.31)> FSBN (0.16)>EBN (0.15). It was far more interesting to further analyze one trait between environments. For example, the correlations of FFSH ranged from 0.16 (FFSH_12KEL and FUHML_12YY) to 0.75 (FFSH_12SHZ and FUHML_13 SHZ). In addition, the average correlation for FFSH in the same site from different years is 0.57 , while the average correlation for FFSH in the same year from different sites is 0.34 (Fig. 2).

\section{Molecular genetic diversity and population structure}

A total of 179 polymorphic markers obtained from the previous study (Nie et al., 2016) were used for genetic analysis, which contained 426 allele loci. The average genetic similarity coefficient variation 
among the 503 cultivars was 0.552 (Nie et al. 2016). The linkage disequilibrium (LD) of this population was analyzed using 179 SSR markers. Based on $r^{2}$ estimates, only $2.09 \%\left(r^{2} \geq 0.05\right)$ and $1.30 \%\left(r^{2} \geq\right.$ 0.1) of the markers showed significant LD, which is be suitable for association mapping (Nie et al., 2016).

Population structure was determined by three methods (PCA plots, Nei's genetic distance, and STRUCTURE software) in the previous study (Nie et al. 2016), according to which, the population were divvied into 7 subgroups.

\section{Association mapping of cotton yield-related traits}

The association analysis was carried out based on the genotypic data, PCA matrix, genetic relationship matrix and BLUP data of ten cotton yield-related traits (Bradbury et al., 2007; Jakobsson and Rosenberg, 2007). Through association analysis, the effects of $G L M(P+G)+Q, G L M(P+G)+P C A$ and $M L M(G+P+Q+K)$ models in association analysis were compared (Fig. S1). For LW and EBN, the above three models had similar effect in controlling population structure; For PH, FFBP, FP and WGP, GLM-Q and MLM-Q-K models were better than GLM-PCA. For SCW, FFSH and FSBN, GLM -PCA model was better than GLM-Q and MLM-Q-K. For LP, GLM-Q model was better than GLM-PCA and MLM-Q-K model. The effect of the MLM-Q$\mathrm{K}$ model in the association analysis is more stable than the other two models, and the effect of the 10 yield-related traits in controlling population structure is smaller. According to the association analysis of the population structure of the above 10 traits under the control of three models, the MLM-Q-K model was suitable for controlling the population structure of ten yield-related traits, and the comprehensive evaluation effect was better than the other two models.

After filtering the minimum alleles ( $\leq 5 \%), 179$ polymorphic SSR markers ( 426 alleles) were used for association analysis based on MLM-Q-K model. At the P $\leq 0.05$ and $P \leq 0.01$ levels, 140 (78.21\%) and 45 (25.14\%) markers were associated with yield-related traits, respectively (Table S3). An average of 5.4 associated markers were detected on each chromosome, (ranging from 1 to 11), with the maximum of 11 markers on chromosomes D05 (Fig. 3). At P $\leq 0.05$ level, the phenotypic variation interpretation rate (PVE) of the QTLs ranged from $0.48 \%$ (NAU4884a and NAU3468a) to 3.89\% (NAU3377db) with an average of $1.30 \%$, while at P $\leq 0.01$ level, the PVE ranged from 1.03\% (MON-CGR5866a) to 3.89\% (NAU3377db), with an average of $2.14 \%$ (Table S3). In addition, one marker was generally associated with multiple traits (Fig. 3). For example, NAU2095 (chromosome A01) was associated with FFSH, PH, FSBN, WGP, LP, and EBN; MON_DC40013 (Chromosome A01) was associated with LW, PH, FFSH, FFBP, and LP; NAU2564 (Chromosome A07) was associated with FFSH, FSBN, and LW; MON_CGR5113 (Chromosome A11) was associated with PH, WGP, FFSH, FSBN, FP, LW, EBN, SCW, and LP; HAU0211 (Chromosome A12) was associated with FFSH, FFBP, FP, LW, EBN, and LP; HAU4022 (Chromosome D02) was associated with PH, FFSH, FFBP, WGP, and FP at an extremely significant level ( $P<0.01$ ); HAU1355 (Chromosome D13) was simultaneously associated with PH, FSBN, and LW; NAU3084 (Chromosome D12) was associated with PH, FFSH, SCW, LW, and EBN (Fig. 3, Table S3). 
LP was associated with most loci among the 10 yield-related traits, with a number of $84(P<0.05)$ and 11 $(P<0.01$ ). PVE ranged from 0.66\% (MON-CGR6410a and BNL1652a) to 3.55\% (NAU2671a), with an average of $1.32 \%(P<0.05)$ and $2.49(P<0.01)$ (Table S3).

FFSH was associated with least loci among the 10 yield-related traits, which ranged from $5(P<0.01)$ to 39 $(\mathrm{P}<0.05)$. The PVE ranged from 0.65\% (NAU4884b) to 3.50\% (HAU4022b) (Table S3).

The number of loci associated with LW was detected in the range of $20(P<0.01)$ to $81(P<0.05)$, and the PVE ranged from $0.48 \%$ (NAU4884a) to $2.54 \%$ (MON-CGR5113c), with an average of $1.12 \%$ (Table S3).

There were $50(P<0.05)$, and $21(P<0.01)$ loci associated with FP. The PVE ranged from 1.01\% (NAU4884b) to $3.61 \%$ (HAU1434b), with an average of $1.83 \%$ (Table S3).

There were $48(P<0.05)$ and $1(P<0.01)$ loci associated with SCW. The PVE ranged from $0.73 \%$ (NAU3904a) to $2.89 \%$ (MON-CGR5167b) (Table S3).

There were $55(P<0.05)$ and $2(P<0.01)$ loci associated with EBN. The PVE ranged from 0.6\% (MON-SHIN1343a) to $2.71 \%$ (NAU5323a) (Table S3).

There were $49(P<0.05)$ and $13(P<0.01)$ loci associated with $\mathrm{PH}$. The PVE ranged from $0.67 \%$ (NAU3607a) to 3.89\% (NAU3377db) (Table S3).

There were 56 (only at $\mathrm{P}<0.05$ ) loci associated with FSBN. The PVE ranged from $0.67 \%(\mathrm{HAU} 1430 \mathrm{~b})$ to 2.49\% (MON-SHIN-0598a) (Table S3).

There were $59(\mathrm{P}<0.05)$ and $7(\mathrm{P}<0.01)$ loci associated with FFBP. The PVE ranged from $0.58 \%(\mathrm{MON}-$ CGR5423d) to $2.29 \%$ (DPL0679a) (Table S3).

There were $46(P<0.05)$ and $14(P<0.01)$ loci associated with WGP. The PVE ranged from $0.48 \%$ (NAU3468a) to 3.39\% (MON_DPL0754a) (Table S3).

\section{Effect value analysis of QTLs associated with yield traits in four breeding stages}

According to the history of cotton breeding stages in China, the 503 upland cotton germplasm resources were divided into 4 groups (Table S4), from breeding stage1 (BS1) to breeding stage 4 (BS4), which represented abroad variation in each experiment site. The ten cotton yield related traits were compared among the 4 breeding stages. According to the results, the BS4 almost represented the highest level of 9 traits, except WGP, which showed a decreased trend during the breeding process; LW, LP, PH, and FFSH showed an increasing trend from BS1 to BS4, while the others showed little changes or random fluctuations during the four breeding stages (Fig. 4).

To further uncover the effect of QTLs on the ten cotton yield-related traits, the phenotypic effect value of each QTL locus in each breeding stage was assessed, which was shown in Fig. 5. For the 48 QTLs of SCW, the average phenotypic effect value of the allele loci in each stage (BS1-BS4) was $-0.11,-0.003$, 
$0.01,-0.003$, respectively (Table S5). The phenotypic effect range of each locus in each stage was -4.96 to $0.31,-0.44$ to $0.38,-0.37$ to 0.24 , and -0.21 to 0.27 , respectively (Table S5). As shown in Fig. 5 a, the phenotypic effect showed an upward trend during the four breeding stages in the marker loci of HAU0590a (-0.09 to 0.02), NAU6966ba (-0.03 to 0.04), and NAU3607a (-4.96 to 0.27), while NAU2858a $(-0.08$ to 0.05$)$ showed a downward trend (Fig. 5a, Table S5).

For the 81 QTLs of LW, the average phenotypic effect value in each stage (BS1-BS4) was $-0.10,-0.01$, $-0.02,-0.01$. The phenotypic effect range of each locus in each stage was -1.76 to $0.26,-0.51$ to $0.12,-1.89$ to 0.10 , and -0.32 to 0.14 , respectively (Table S5). As shown in Fig. $5 b$, the phenotypic effects showed an upward trend in the following loci: NBRI_HQ524733d (-0.13 to 0.01), NAU3607a (-1.76 to 0.14), NBRI_HQ524733c (-0.14 to 0.01), MON_CGR5399c (0.01 to 0.07) and NAU3084c (-0.22 to-0.01). HAU2770a (0.01 to 0.06), NBRI_HQ524733a (-0.001 to 0.04) and NAU3774a (0.003 to 0.08), showed a downward trend (Fig. 5b, Table S5).

For the 84 alleles of $L P$, the average phenotypic effect value in each stage was $-0.6854,-0.23,-0.04,-0.04$. The phenotypic effect range of each locus in each stage was -1.57 to $3.00,-8.80$ to $2.93,-3.85$ to 4.94 , and -2.70 to 4.35 , respectively (Table S5). As shown in Fig. 5c, the phenotypic effects of the four breeding stages showed an upward trend in the following loci: HAU0197b (-1.58 to 0.28), HAU0083c ( -5.15 to -0.05), HAU3812a (-0.40 to 0.38), NAU2095a (-2.01 to 0.44), and MON_CGR6356b (-3.04 to -1.03$)$. MON_CGR6356a (0.0003 to 0.44$)$, MON_CGR6472a (0.025 to 1.45$)$, NAU3774a (0.04 to 2.29 ), and HAU0197a (-0.001 to 0.61) showed a decreasing trend (Fig. 5c, Table S5).

For the 55 QTLs of EBN, the average phenotypic effect value in each stage was $-0.28,0.05,-0.02$ and -0.02 , with the variation range of -2.99 to $1.74,-1.37$ to $2.29,-1.12$ to 1.39 and -2.33 to 1.54 , respectively (Table S5). As shown in Fig. 5d, the phenotypic effects showed an upward trend in MON_CGR5867a (-0.91 to 0.34$)$, MON_CGR6378c (-2.99 to 0.81$)$, HAU4552a (-0.31 to 0.09$),$ HAU0119c (-1.19 to 0.45$)$, and HAU1382a (-0.92 to -0.08$)$. CCRI192a (-1.06 to 0.17$)$ and HAU3966b $(-0.22$ to -0.03$)$ showed a decreasing trend (Fig. 5d, Table S5).

Among the QTLs of PH, the phenotypic effect of DPL0475a showed an upward trend, while MON_CGR5167b and BNL3790c showed downward trend (Fig. 5e). Among the QTLs of FFSH, the phenotypic effect of HAU0211c showed an upward trend, while MON_CGR5423e showed downward trend (Fig. 5f). Among the QTLs of FSBN, the phenotypic effect of BNL3347a and MON_CGR5867c showed an upward trend, while HAU2835b, HAU1355a, MON_CGR5866b, HAU0197a, HAU1434b and DPL0461c showed downward trend (Fig. $5 \mathrm{~g}$ ). Among the QTLs of FFBP, the phenotypic effect of MON_DPL0544e showed an upward trend, while MON_CGR5866a and CK98221_PR_SSc showed downward trend (Fig. 5h). Among the QTLs of FP, the phenotypic effect of MON_DPL0544e, MON_CGR5113e, HAU4022b, MON_CGR5399c, and DPL0475b, showed increased from BS1 to BS4, while NAU3468a and NAU3966b decreased (Fig. 5i). Among the QTLs of WGP, the phenotypic effect of MON_DPL0544e increased from BS1 to BS4, while phenotypic effect of MON_CGR5866a, NAU2858a and NAU3966b decreased. 


\section{Accumulation of favorable alleles for major QTL in four cotton breeding stages and typical carrier materials}

9 major QTLs $\left(R^{2} \geq 3\right)$ were used for accumulation of favorable alleles analysis, including three QTLs for LP (NAU2671, NAU3774, and MON-CGR6356), three QTLs for PH (NAU3377, MON-CGR5113, and NAU2095), one QTL for FFSH (HAU4022), one QTL for FP (HAU1434), and one QTL for WGP (HAU1434). NAU2671 has three alleles, including NAU2671a, NAU2671b, and NAU2671e, among which NAU2671a was the favorable allele (Fig. 6a), showing the highest FS. The frequency of NAU2671a showed an increasing trend during the four breeding stages (0.62 to 0.79) (Fig. 6a). NAU3774 has three alleles, including NAU3774a, NAU3774b, and NAU3774d, among which, NAU3774a was the favorable allele. The frequency of NAU3774a showed an increasing trend during the four breeding stages ( 0.19 to 0.70$)$ (Fig. 6b). MON-CGR6356 had three alleles, including MON-CGR6356a, MON-CGR6356b, and MON-CGR6356c, among which, MON-CGR6356a was the favorable allele. The frequency of MON-CGR6356a showed an increasing trend during the four breeding stages (0.71 to 0.92) (Fig. 6c). NAU3377c/d are the favorable alleles of PH, which could increase the plant height. The frequency of NAU3377c/d showed a certain fluctuation, and the frequencies of BS3 and BS4 were slightly decreased (Fig. 6d). MON-CGR5113a/d were favorable alleles of PH. The frequency of MON-CGR5113a/d showed an increasing trend from BS1 to BS3, while showed a little decrease in BS4 (Fig. 6e). NAU2095a is a favorable allele of PH, and the frequency of which showed an irregular fluctuation among the 4 breeding stages, while the frequencies of BS3 and BS4 were slightly increased. HAU4022a/c were favorable alleles of FFSH, the frequency of which was relatively stable among the 4 breeding. HAU1434a/c were favorable alleles of both FP and WGP, which showed an increasing trend from BS1 to BS4 (0.80 to 0.95) (Fig. 6i).

Additionally, the typical carrier materials possessing the favorable allele were shown in table S6. For LP, the umber carrier materials with the three favorable alleles (NAU2671a, NAU3774a, and MON-CGR6356a) was 136 , such as $Z Y 1, Z Y 27, Z Y 28$, and $Z Y 44$. For PH, there were 19 materials carried the three favorable alleles (NAU3377c/d, MON-CGR5113a/d, and NAU2095a), such as ZY13, ZY55 and ZY71. For FFSH, there were 473 materials carrying the favorable alleles HAU4022a/c. For FP and WGP, there were 429 materials carrying the favorable alleles HAU1434a/c. However, there were only three materials carrying the 8 favorable alleles, including ZY92, ZY398, and ZY87 (Table S6). ZY92 was derived from the BS3, while ZY398 and ZY87 were derived from BS4.

\section{Candidate gene annotation and prediction}

Four artificially selected major QTL during the breeding process, including three QTL of LP (NAU2671, NAU3774, and MON-CGR6356), one QTL of FP and WGP (HAU1434), were used for candidate gene annotation and prediction. Candidate genes were listed within the candidate regions of QTLs, which were set around the LD decay distance as $400 \mathrm{~kb}$. There were 34, 42, 20 and 22 candidate genes for the 4 QTL (NAU2671, NAU3774, MON-CGR6356, and HAU1434) respectively (Table S7). One key candidate gene for each QTL was predicted according to the functional annotation. For NAU2671 of LP, Ghir_A12G024420 encoded a homeobox protein BEL1 was expressed in all tested tissues (Fig. 7a); in Arabidopsis, BEL1- 
LIKE HOMEODOMAIN6 was involved in secondary cell wall formation of interfascicular fiber (Liu et al. 2014). For NAU3774 of LP, Ghir_D12G026070 encoded a Cytochrome B5 protein, which was highly expressed in the developing fibers (Fig. 7b); in Arabidopsis, Cytochrome b 5 worked as an obligate electron shuttle for syringyl lignin biosynthesis, which also played an important role in the cotton fiber development (Gou et al. 2019). For MON-CGR6356 of LP, Ghir_A01G016840 encoded auxin-induced 5NG4 protein, which was expressed specially in the developing fibers (Fig. 7c); auxin-induced protein 5NG4 was associated with culm cellulose content in bread wheat (Kaur et al. 2017). For HAU1434 of FP and WGP, Ghir_A12G013050 encoded a SBP domain protein, which was highly expressed specially in the floral organ (Fig. 7d); SBP domain proteins were widely reported to be involved in flowering initiation and flower development (Cardon et al. 1997; Hou et al. 2017; Yamasaki et al. 2006).

\section{Discussion}

\section{Extensive genetic variation and multiple environments contribute to QTL mapping}

Genome-wide association study using natural population is a powerful strategy to effectively fine map QTL due to a great number of historical recombination events that lead to the rapid decay of linkage disequilibrium (LD) (Flint-Garcia et al. 2003; Li et al. 2013). The population size and diversity of germplasm resources could affect the LD and resolution of QTL. In our study, 503 germplasms derived from United States, the former Soviet Union, and China were divvied into 7 subgroups by using the SSR markers, which represents extensive genetic variations and suitable for GWAS. In addition, quantitative traits are generally susceptible to environmental conditions, so the QTLs stable in multiple environments were more reliable, which could be used for gene cloning and marker assistance selection (MAS)(Raihan et al. 2016; Wang et al. 2015). In the study, the data of 5 traits were collected from 8 environments (4 typical cotton growing areas in China; 2 years), and BLUP phenotypic data were used to provide comprehensive multi-environmental accurate phenotypic values for association analysis. According to the results, the extensive genetic variation and multiple environments of the study make the QTLs more reliable.

In order to further verify the reliability of the association mapping results, the QTLs were compared to previous study. As a result, some of the QTLs were consistent with previous studies. For example, an LP and SCW related loci, BNL3590 (Chr A02), has been reported in two studies, where it was associated with SCW (An et al. 2010; Mei et al. 2013). MON_CGR5399 (Chr A10) has been reported to be associated with LW in previous study, while, in the study, MON_CGR5399 was associated with multiple traits, including LW, FP, PH, FFBP and SCW(Wang et al. 2015). NAU3377 (Chr D11) been reported to be associated with LP in the previous study, while in the study (Wang et al. 2007), NAU3377 (Chr D11) was not only associated with LP, but also EBN, LW, PH, SCW. NAU3774 (Chr D12) been reported to be associated with LW, while, in the study, NAU3774 was associated with both LW and LP (Wang et al. 2015). Additionally, there were more new QTLs identified in the study, which could be used for further genetic analysis of cotton yieldrelated traits. 


\section{The artificial selection during the breeding process contributes to the increased cotton production}

As a natural fiber resource, Gossypium was cultivated about 7000 years ago (Fang et al. 2017a). During the process of cultivation, its fiber productivity has been increased duo to natural genome polyploidization and artificial selection (Jiang et al. 1998; Yuan et al. 2015). Artificial selection could influence the allele frequency through the selection of preferred traits in the traditional breeding process (Luikart et al. 2003). Mutation and recombination are two important factors in determining the efficiency of selection (Nachman and Payseur, 2012; Noor and Bennett, 2009). The comparison of the genome sequence of Gossypium hirsutum CRI-12 family (including CRI-12, its parental cultivars, and progeny cultivars) revealed that 1029 haplotype blocks may be recombined under artificial selection (Lu et al. 2019). Strong artificial selection during domestication has resulted in reduced genetic diversity but stronger linkage disequilibrium and higher extents of selective sweeps (Ma and Wang, 2019). In our study, LW, LP, PH, and FFSH showed significant increasing trends during the cotton breeding process, while WGP showed a significant decreased trend, which was just consistent with the evolution of cotton varieties in Xinjiang of China, and resulted in higher yield but shorter growing period cotton varieties. The effect and evolution analysis of QTLs associated with yield traits in four breeding stages have been assessed. 29 QTLs showed an increasing trend contribution to cotton yield-related traits from BS1 to BS4, which could be caused by increasing favorable allele frequency during artificial selection breeding process. However, 26 QTLs showed decreased trend effect from BS1 to BS4, which showed that there was a great potential to gain cotton yield by increasing the favorable alleles of those QTLs. Additionally, the favorable allele frequency of major QTL were detected, and three favorable allele associated with FP (NAU2671a, NAU3774a, and MON-CGR6356a) were strongly selected during the cotton breeding process, which contributed to the increasing FS of cotton; one favorable allele associated with WGP were strongly selected, which contributed the decreasing WGP of cotton. According to the results, cotton breeding process is a genetic improvement to increase frequency and number of favorable alleles. However, there were still some favorable alleles were not affected or negative selected during the evolution, which could be as potential genetic resource for cotton genetic improvement in the future. from BS1 to BS4,

\section{The QTLs identified in the study lay the foundation for gene cloning and molecular breeding}

With the developing of genome sequencing technology, the reference genome sequences of Gossypium hirsutum have been well developed (Wang et al. 2019), which provide lots of useful information for candidate gene identification from the results of GWAS (Huang et al. 2018; Nie et al. 2020). According to the reference genome, the SSR markers significantly associated with cotton-yield related traits could be anchored on physical maps, which provided candidate regions based on LD decay distance (Huang et al. 2018). The annotated genes in the regions could be used for further verification by functional annotation or using reverse genetics methods (Fang et al. 2017b; Li et al. 2017). In our study, at the level p $\leq 0.01,45$ QTLs were identified to be associated with cotton yield related traits, among which, 8 major QTLs were used for favorable allele identification. Additionally, the materials which carried the favorable alleles were identified, which could be used as donors to improve the cotton yield related traits, such as ZY92, ZY398, and ZY87, which carried 8 favorable alleles. Four strongly selected alleles were used for candidate

Page 12/23 
predication according to gene functional annotation and tissue expression. As a result, three candidate genes were identified for LP, which were associated with fiber development according to the function of homologous in Arabidopsis and wheat (Gou et al. 2019; Kaur et al. 2017; Liu et al. 2014). One candidate gene was identified for FS and WGP, which was specifically expressed in flower organs, and the homologous in other plants were involved in flowering initiation and flower development (Cardon et al. 1997; Yamasaki et al. 2006).

\section{Conclusion}

In this study, 140 QTL loci significantly associated with ten fiber yield related traits were identified by using 503 upland cotton varieties covering the 4 breeding stages in China, among which, 29 QTL loci showed an increasing trend contribution to cotton yield-related traits from BS1 to BS4, and 26 QTL loci showed decreased trend effect. 4 favorable alleles of 9 major QTLs (R2 $\geq 3)$ were strongly selected during the breeding stages, and the candidate genes of the 4 strongly selected alleles were predicated according to the gene function annotation and tissue expression data. The study not only uncovers the genetic basis of 10 cotton yield related traits, but also provides genetic evidence for cotton improvement during the cotton breeding process in China.

\section{Declarations}

\section{Acknowledgements}

We would like to thank the anonymous reviewers for their valuable comments and helpful suggestions which help to improve the manuscript.

\section{Authors' contributions}

NX, LZ and PZ designed the experiments. GC, PZ, NX, YC, HC, SC, ZX and ZR performed the experiments. NX, GC and PZ wrote the main manuscript text and prepared all Fig.s. GC, PZ, HC, SC and YQ performed data analysis. NX, PZ, LZ and ZL revised and polished the manuscript. All authors contributed in the interpretation of results and approved the final manuscript.

\section{Funding}

This work was supported by the National Natural Science Foundation of China (31760402), Young and middle-aged science and technology leading talents of Xinjiang Production And Construction Corps (2019CB027).

\section{Availability of data and materials}

The datasets used and analyzed during the current study are available from the corresponding author on reasonable request. 


\section{Ethics approval and consent to participate}

Not applicable

\section{Consent for publication}

All Authors have provided ethical approval and consent to participate as well as consent for publication.

\section{Competing interests}

The authors have declared that no competing interests exist.

\section{Author details}

a Key Laboratory of Oasis Ecology Agricultural of Xinjiang Bingtuan, Agricultural College, Shihezi University, Shihezi, Xinjiang, 832003, China. ${ }^{b}$ Cotton Research Institute, Shihezi Academy of Agriculture Science, Shihezi, 832011, Xinjiang, China. ' Cotton Institute, Xinjiang Academy of Agriculture and Reclamation Science, Shihezi 832000, Xinjiang, China. dNational Key Laboratory of Crop Genetic Improvement, College of Plant Sciences \& Technology, Huazhong Agricultural University, Wuhan, 430070, Hubei, China. ${ }^{e} H u b e i$ Key Laboratory of Agricultural Bioinformatics, College of Informatics, Huazhong Agricultural University, Wuhan 430070, Hubei, China.

\section{References}

Ademe MS, He SP, Pan ZE, et al. Association mapping analysis of fiber yield and quality traits in Upland cotton (Gossypium hirsutum L.). Mol Genet Genomics. 2017; 292: 1267-1280. doi: 10.1007/s00438-0171346-9.

An CF, Jenkins JN, Wu JX, et al. Use of fiber and fuzz mutants to detect QTL for yield components, seed, and fiber traits of upland cotton.2010; Euphytica. 172, 21-34. https://doi.org/10.1007/s10681-009-0009-2

Benjamini Y, Hochberg Y. Controlling the False Discovery Rate: a Practical and Powerful Approach to Multiple Testing. Journal of the royal statistical society. 1995; 57, 289-300.

Bradbury PJ, Zhang Z, Kroon DE, et al. TASSEL: software for association mapping of complex traits in diverse samples. Bioinformatics.2007; 23, 2633-2635. doi: 10.1093/bioinformatics/btm308.

Cardon GH, Höhmann S, Nettesheim K, et al. Functional analysis of the Arabidopsis thaliana SBP-box gene SPL3: a novel gene involved in the floral transition. Plant J. 1997; 12, 367-377. doi: 10.1046/j.1365313x.1997.12020367.x

Chen ZJ, Scheffler BE, Dennis E, et al. Toward sequencing cotton (Gossypium) genomes. Plant Physiol. 2007; 145, 1303-1310. doi: 10.1104/pp.107.107672. 
Dong CG, Wang J, Chen QJ, et al. Detection of favorable alleles for yield and yield components by association mapping in upland cotton. Genes Genomics. 2018; 40, 725-734. doi: 10.1007/s13258-0180678-0.

Du XM, Huang G, He SP, et al. Resequencing of 243 diploid cotton accessions based on an updated A genome identifies the genetic basis of key agronomic traits. Nat Genet. 2018; 50, 796-802. doi: 10.1038/s41588-018-0116-x.

Fang L, Gong H, Hu Y, et al. Genomic insights into divergence and dual domestication of cultivated allotetraploid cottons. Genome biology. 2017a; 18, 33-33. doi: 10.1186/s13059-017-1167-5.

Fang L, Wang Q, Hu Y, et al. Genomic analyses in cotton identify signatures of selection and loci associated with fiber quality and yield traits. Nat Genet. 2017b; 49, 1089-1098. doi: 10.1038/ng.3887.

Flint-Garcia SA, Thornsberry JM, Buckler ESt. Structure of linkage disequilibrium in plants. Annu Rev Plant Biol. 2003; 54, 357-374. doi: 10.1146/annurev.arplant.54.031902.134907.

Gore MA, Fang DD, Poland J, et al. Linkage Map Construction and Quantitative Trait Locus Analysis of Agronomic and Fiber Quality Traits in Cotton. The Plant Genome. 2014; 7(1):1-10. doi: 10.3835/plantgenome2013.07.0023

Gou MY, Yang XM, Zhao YJ, et al. Cytochrome b5 is an obligate electron shuttle protein for syringyl lignin biosynthesis in Arabidopsis. Plant Cell. 2019; 31, 1344-1366. doi: 10.1105/tpc.18.00778.

Hou H, Yan X, Sha T, et al. The SBP-Box Gene VpSBP11 from Chinese Wild Vitis Is Involved in Floral Transition and Affects Leaf Development. Int J Mol Sci.2017; 18(7):1493. doi: 10.3390/ijms18071493.

Huang C, Shen C, Wen TW,et al. SSR-based association mapping of fiber quality in upland cotton using an eight-way MAGIC population. Mol Genet Genomics.2018; 293, 793-805. doi: 10.1007/s00438-0181419-4.

Huang XH, Yang SH, Gong JY, et al. 2016. Genomic architecture of heterosis for yield traits in rice. Nature.2016; 537, 629-633. doi: 10.1038/nature19760.

Hufford MB, Xu X, van Heerwaarden J, et al. Comparative population genomics of maize domestication and improvement. Nat Genet.2012; 44, 808-811. doi: 10.1038/ng.2309.

Jakobsson M, Rosenberg NA. CLUMPP: a cluster matching and permutation program for dealing with label switching and multimodality in analysis of population structure. Bioinformatics. 2007; 23, 18011806. doi: 10.1093/bioinformatics/btm233.

Jia YH, Sun XW, Sun JL,et al. 2014. Association mapping for epistasis and environmental interaction of yield traits in 323 cotton cultivars under 9 different environments. PLoS One.2014; 9, e95882. doi: 10.1371/journal.pone.0095882. 
Jiang C, Wright RJ, El-Zik KM,et al. Polyploid formation created unique avenues for response to selection in Gossypium (cotton). Proc Natl Acad Sci U S A.1998; 95, 4419-4424. doi: 10.1073/pnas.95.8.4419.

Kaur S, Zhang X, Mohan A, et al. Genome-Wide Association Study Reveals Novel Genes Associated with Culm Cellulose Content in Bread Wheat (Triticum aestivum, L.). Front Plant Sci. 2017; 8, 1913. doi: 10.3389/fpls.2017.01913.

Li C, Zhao TL, Yu HR, et al. Genetic basis of heterosis for yield and yield components explored by QTL mapping across four genetic populations in upland cotton. BMC Genomics. 2018a; 19, 910. doi: 10.1186/s12864-018-5289-2.

Li Fj, Wen We, He Zh, et al. Genome-wide linkage mapping of yield-related traits in three Chinese bread wheat populations using high-density SNP markers. Theor Appl Genet. 2018b; 131, 1903-1924. doi: 10.1007/s00122-018-3122-6.

Li H, Peng ZY, Yang XH, et al. Genome-wide association study dissects the genetic architecture of oil biosynthesis in maize kernels. Nat Genet. 2013; 45, 43-50. doi: 10.1038/ng.2484.

Li TG, Ma XF, Li NY, et al. Genome-wide association study discovered candidate genes of Verticillium wilt resistance in upland cotton (Gossypium hirsutum L.). Plant Biotechnol J. 2017; 15, 1520-1532. doi: 10.1111/pbi.12734.

Liu RZ, Wang BH, Guo WZ, et al. Quantitative trait loci mapping for yield and its components by using two immortalized populations of a heterotic hybrid in Gossypium hirsutum L. Molecular Breeding. 2012; 29, 297-311. https://doi.org/10.1007/s11032-011-9547-0

Liu YY, You SJ, Taylor-Teeples M, et al. BEL1-LIKE HOMEODOMAIN6 and KNOTTED ARABIDOPSIS THALIANA7 interact and regulate secondary cell wall formation via repression of REVOLUTA. Plant Cell. 2014; 26, 4843-4861. doi: 10.1105/tpc.114.128322.

Lu XK, Fu XQ, Wang DL, et al. Resequencing of cv CRI-12 family reveals haplotype block inheritance and recombination of agronomically important genes in artificial selection. Plant Biotechnol J.2019; 17, 945955. doi: 10.1111/pbi.13030.

Luikart G, England PR, Tallmon DA,et al. The power and promise of population genomics: from genotyping to genome typing. Nature Reviews Genetics. 2003; 4, 981-994. doi: 10.1038/nrg1226.

Ma XF, Wang ZY Li W, et al. Resequencing core accessions of a pedigree identifies derivation of genomic segments and key agronomic trait loci during cotton improvement. Plant Biotechnol J. 2019; 17, 762-775. doi: 10.1111/pbi.13013.

Maik W, Abid MA, Cheema HM, et al. FROM Qutn TO Bt COTTON: DEVELOPMENT, ADOPTION AND PROSPECTS. A REVIEW. Tsitol Genet.2015; 49, 73-85. 
Mei HX, Zhu XF, Zhang TZ. Favorable QTL Alleles for Yield and Its Components Identified by Association Mapping in Chinese Upland Cotton Cultivars. PLoS One.2013; 8(12):e82193. doi:

10.1371/journal.pone.0082193.

Mengistu DK, Kidane YG, Catellani M, et al. High-density molecular characterization and association mapping in Ethiopian durum wheat landraces reveals high diversity and potential for wheat breeding. Plant Biotechnol J. 2016; 14, 1800-1812. doi: 10.1111/pbi.12538.

Nachman MW, Payseur BA. Recombination rate variation and speciation: theoretical predictions and empirical results from rabbits and mice. Philos Trans R Soc Lond B Biol Sci. 2012; 367, 409-421. doi: 10.1098/rstb.2011.0249.

Nie XH, Huang C, You CY, et al. Genome-wide SSR-based association mapping for fiber quality in nationwide upland cotton inbreed cultivars in China. BMC Genomics.2016; 17, 352. doi: 10.1186/s12864-0162662-x.

Nie XH, Wen TW, Shao PX, et al. High-density genetic variation maps reveal the correlation between asymmetric interspecific introgressions and improvement of agronomic traits in Upland and Pima cotton varieties developed in Xinjiang, China. Plant J.2020; 103, 677-689. doi: 10.1111/tpj.14760.

Noor MA, Bennett SM. Islands of speciation or mirages in the desert? Examining the role of restricted recombination in maintaining species. Heredity (Edinb). 2009; 103, 439-444. doi: 10.1038/hdy.2009.151.

Raihan MS, Liu J, Huang J, et al. Multi-environment QTL analysis of grain morphology traits and fine mapping of a kernel-width QTL in Zheng58 × SK maize population. Theor Appl Genet.2016; 129, 14651477. doi: 10.1007/s00122-016-2717-z.

Soltis NE, Atwell S, Shi G, et al. Interactions of Tomato and Botrytis cinerea Genetic Diversity: Parsing the Contributions of Host Differentiation, Domestication, and Pathogen Variation. Plant Cell. 2019; 31, 502519. doi: $10.1105 /$ tpc. 18.00857.

Sun ZW, Wang XF, Liu ZW, et al. A genome-wide association study uncovers novel genomic regions and candidate genes of yield-related traits in upland cotton. Theor Appl Genet. 2018; 131, 2413-2425. doi: $10.1007 / \mathrm{s} 00122-018-3162-\mathrm{y}$.

Wang BH, Guo WZ, Zhu XF, et al. QTL mapping of yield and yield components for elite hybrid derived-RILs in upland cotton. J Genet Genomics. 2007; 34(1):35-45. doi: 016/S1673-8527(07)60005-8.

Wang HT, Huang C, Guo HL, et al. QTL Mapping for Fiber and Yield Traits in Upland Cotton under Multiple Environments. PLoS One. 2015; 10(6):e0130742. doi: 10.1371/journal.pone.0130742.

Wang MJ, Tu LL, Yuan DJ, et al. Reference genome sequences of two cultivated allotetraploid cottons, Gossypium hirsutum and Gossypium barbadense. Nat Genet. 2019, 51, 224-229. doi: 10.1038/s41588018-0282-x. 
Xue S, Bradbury PJ, Casstevens TM, et al. Genetic Architecture of Domestication-Related Traits in Maize. Genetics. 2016; 204, 99-113. doi: 10.1534/genetics.116.191106.

Yamasaki K, Kigawa T, Inoue M, et al. An Arabidopsis SBP-domain fragment with a disrupted C-terminal zinc-binding site retains its tertiary structure. FEBS Lett. 2006; 580, 2109-2116. doi:

10.1016/j.febslet.2006.03.014.

Yang N, Lu YL, Yang XH,et al. Genome wide association studies using a new nonparametric model reveal the genetic architecture of 17 agronomic traits in an enlarged maize association panel. PLoS Genet. 2014; 10(9):e1004573. doi: 10.1371/journal.pgen.1004573.

Yuan DJ, Tang ZH, Wang MJ, et al. The genome sequence of Sea-Island cotton (Gossypium barbadense) provides insights into the allopolyploidization and development of superior spinnable fibres. Sci Rep. 2015; 5, 17662. doi: 10.1038/srep17662.

Zhang D, Zhang HY, Hu ZB, et al. Artificial selection on GmOLEO1 contributes to the increase in seed oil during soybean domestication. PLoS Genet. 2019; 15(7):e1008267. doi: 10.1371/journal.pgen.1008267.

Zhang TZ, Hu Y, Jiang WK, et al. Sequencing of allotetraploid cotton ( Gossypium hirsutum L. acc. TM-1) provides a resource for fiber improvement. Nat Biotechnol, 2015; 33, 531-537. doi: 10.1038/nbt.3207.

Zhang Z, Li JW, Jamshed M, et al. Genome-wide quantitative trait loci reveal the genetic basis of cotton fibre quality and yield-related traits in a Gossypium hirsutum recombinant inbred line population. Plant Biotechnol J. 2020; 18, 239-253. doi: 10.1111/pbi.13191.

Zhao GW, Lian Q, Zhang ZH, et al. A comprehensive genome variation map of melon identifies multiple domestication events and loci influencing agronomic traits. Nat Genet. 2019; 51, 1607-1615. doi:

10.1038/s41588-019-0522-8.

Zheng J, Wu H, Zhu HB, et al. Determining factors, regulation system, and domestication of anthocyanin biosynthesis in rice leaves. New Phytol. 2019; 223, 705-721. doi: 10.1111/nph.15807.

Zhou ZK, Jiang Y, Wang Z, et al. Resequencing 302 wild and cultivated accessions identifies genes related to domestication and improvement in soybean. Nat Biotechnol. 2015; 34, 441-441. doi:

10.1038/nbt.3096.

\section{Figures}



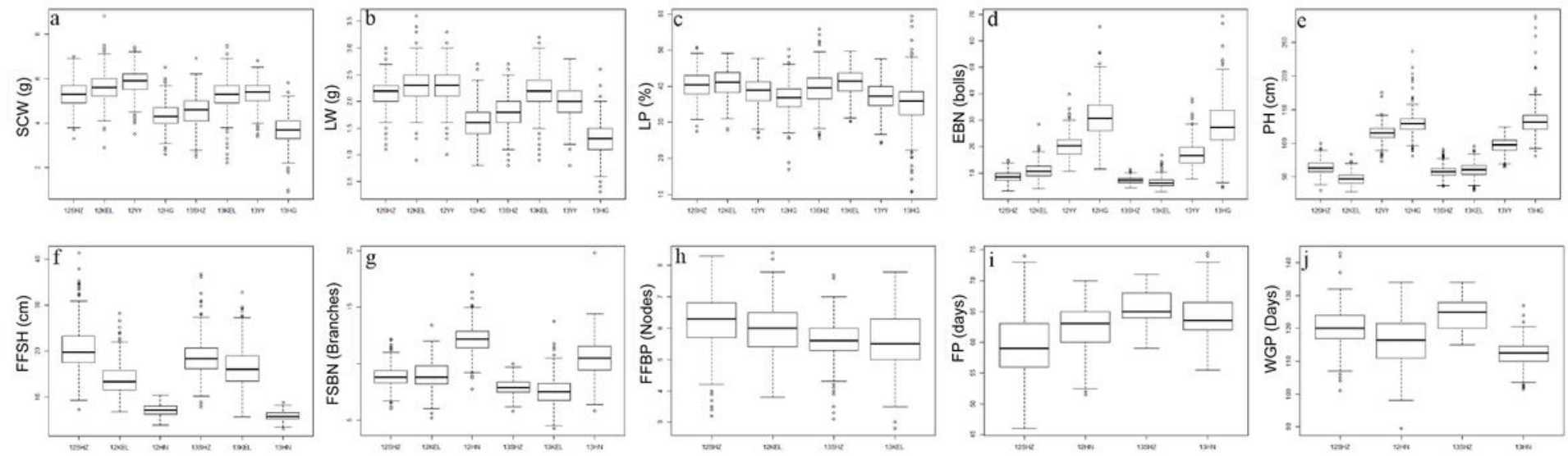

\section{Figure 1}

The boxplots of the changing trends of ten yield-related traits in multiple environments. 12SHZ (Shihezi in 2012), 12KEL (Kuerle in 2012), 12YY (Yuanyang in 2012), 12HG (Huanggang in 2012), 13SHZ (Shihezi in 2013), 13KEL (Kuerle in 2013), 13YY (Yuanyang in 2013), 13HG (Huanggang in 2013).
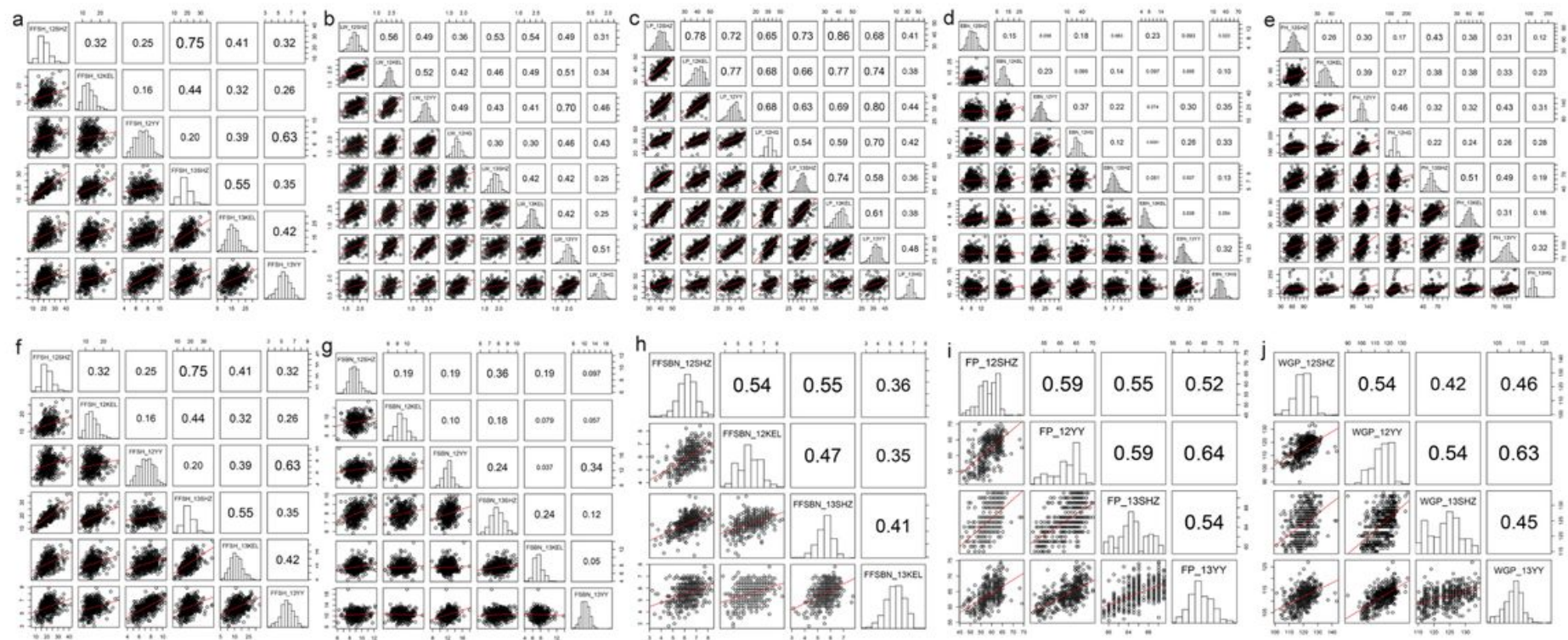

Figure 2

The correlations of ten yield-related traits in multiple environments. a. Correlations of SCW in eight environments. b. Correlations of LW in eight environments. c. Correlations of LP in eight environments. $d$. Correlations of EBN in eight environments. e. Correlations of $\mathrm{PH}$ in eight environments. f. Correlations of FFSH in six environments. g. Correlations of FSBN in six environments. h. Correlations of FSBP in four environments. i. Correlations of FP in four environments. j. Correlations of WGP in four environments. 

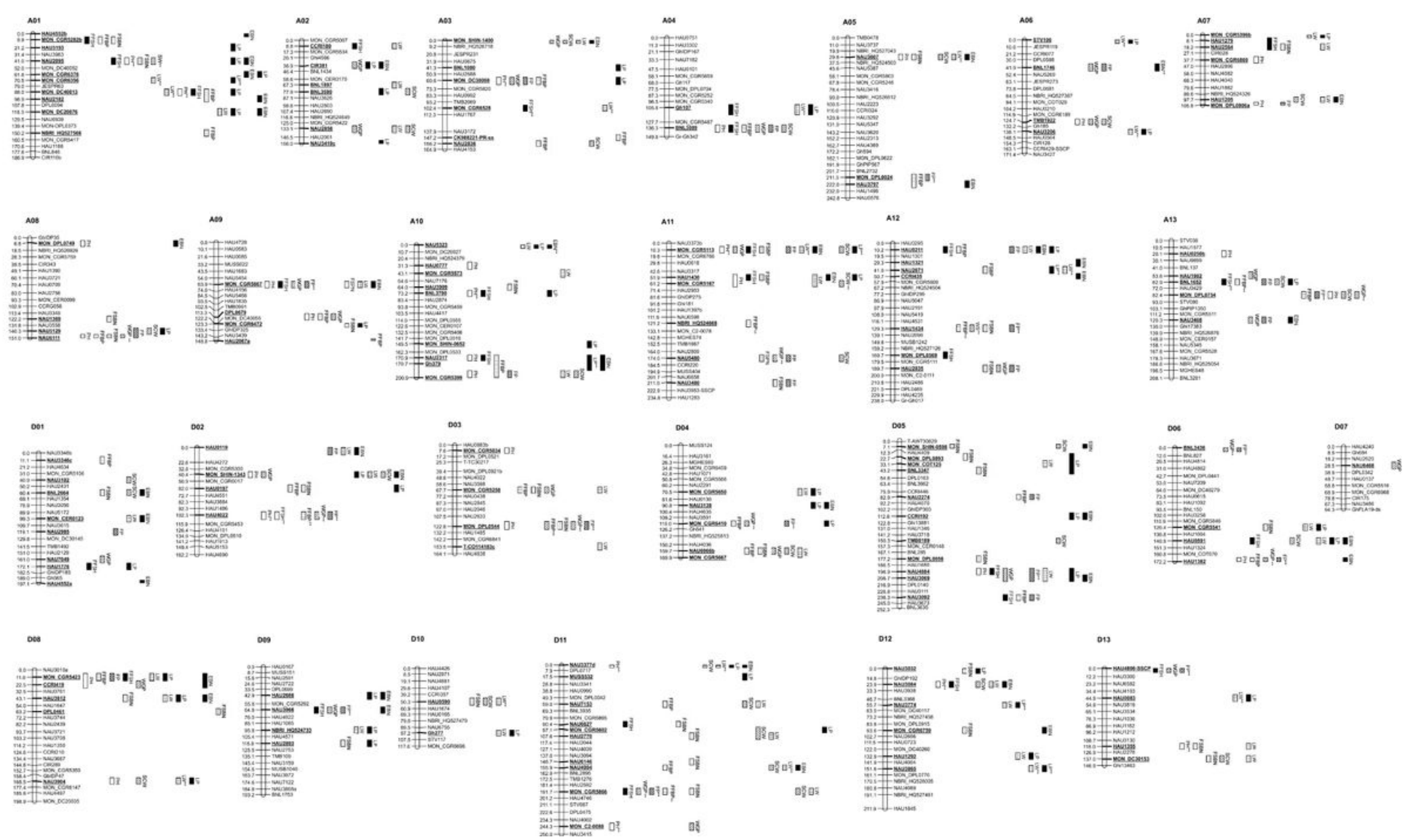

Figure 3

The distribution of the QTLs associated with the yield-related traits on 26 chromosomes. 

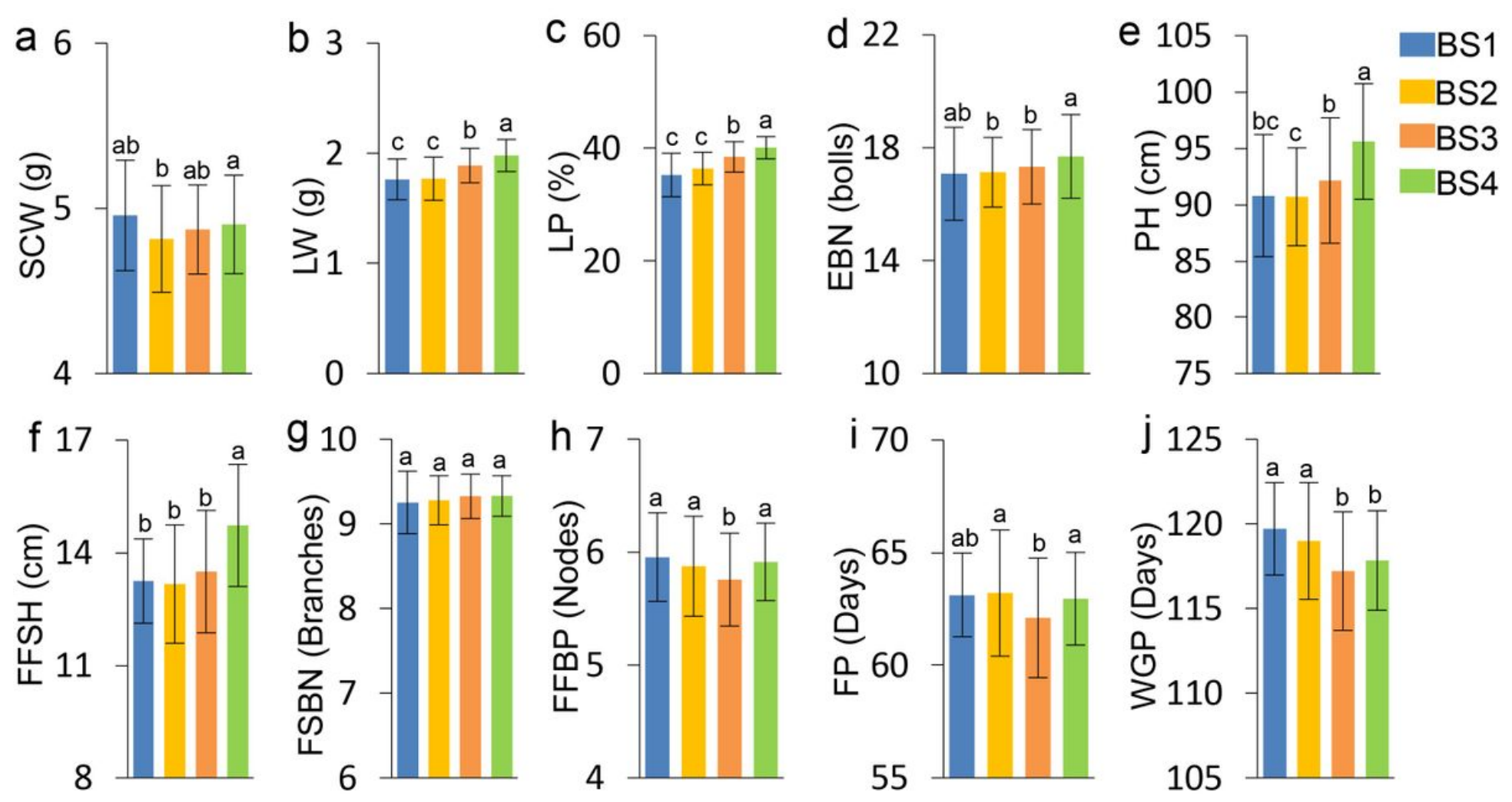

\section{Figure 4}

The statistic analysis and comparison of the ten cotton yield-related traits in the four breeding stages.

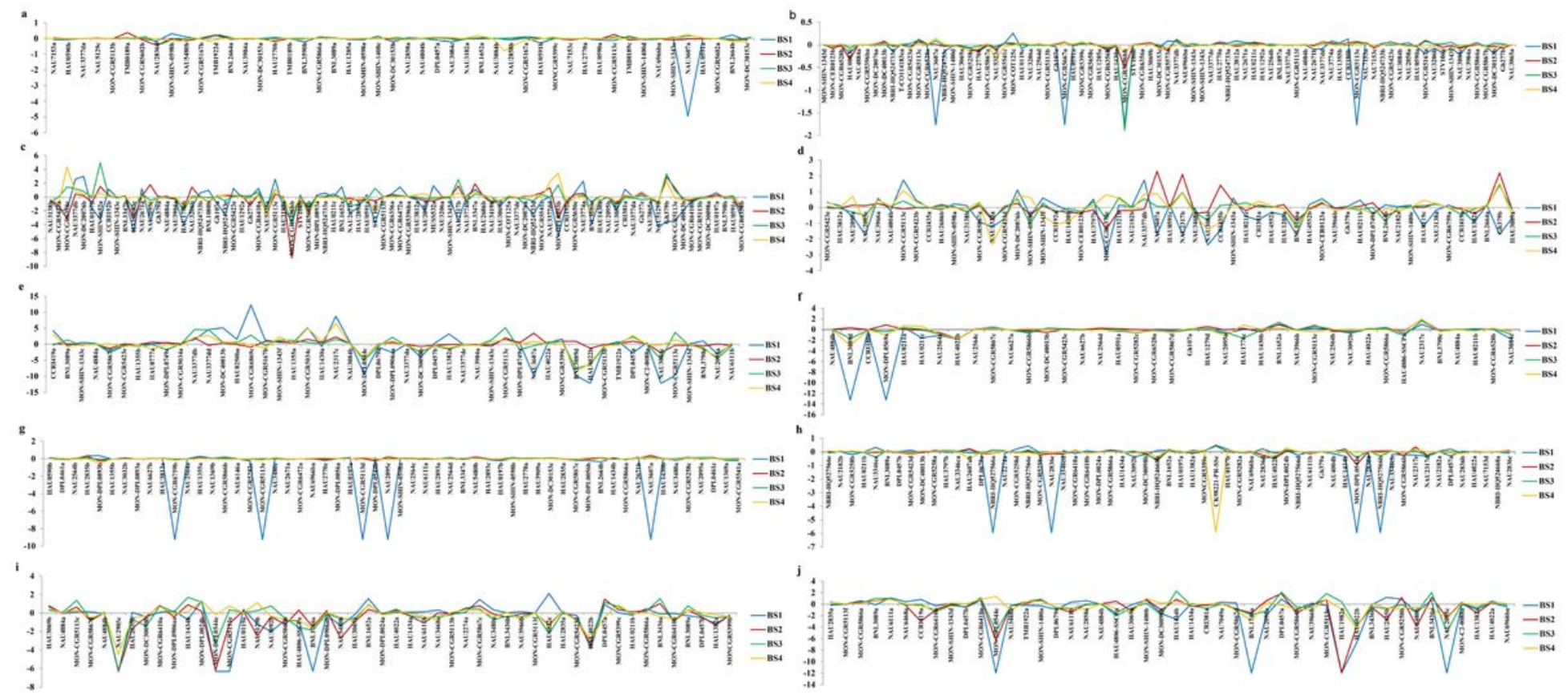

\section{Figure 5}


The change trends of phenotypic effect of marker loci associated with ten yield-related traits in the four breeding stages. a. SCW (seed cotton weight), b. LW (lint weight), c. LP (lint percentage), d. EBN (effective boll number), e. PH (plant height), f. FFSH (first fruit spur height), g. FSBN (fruit spur branch number), h. FFBP (first fruit branch position), i. FP (flowering period), j. WGP (whole growth period). The four different colored lines indicate four different breeding stages.
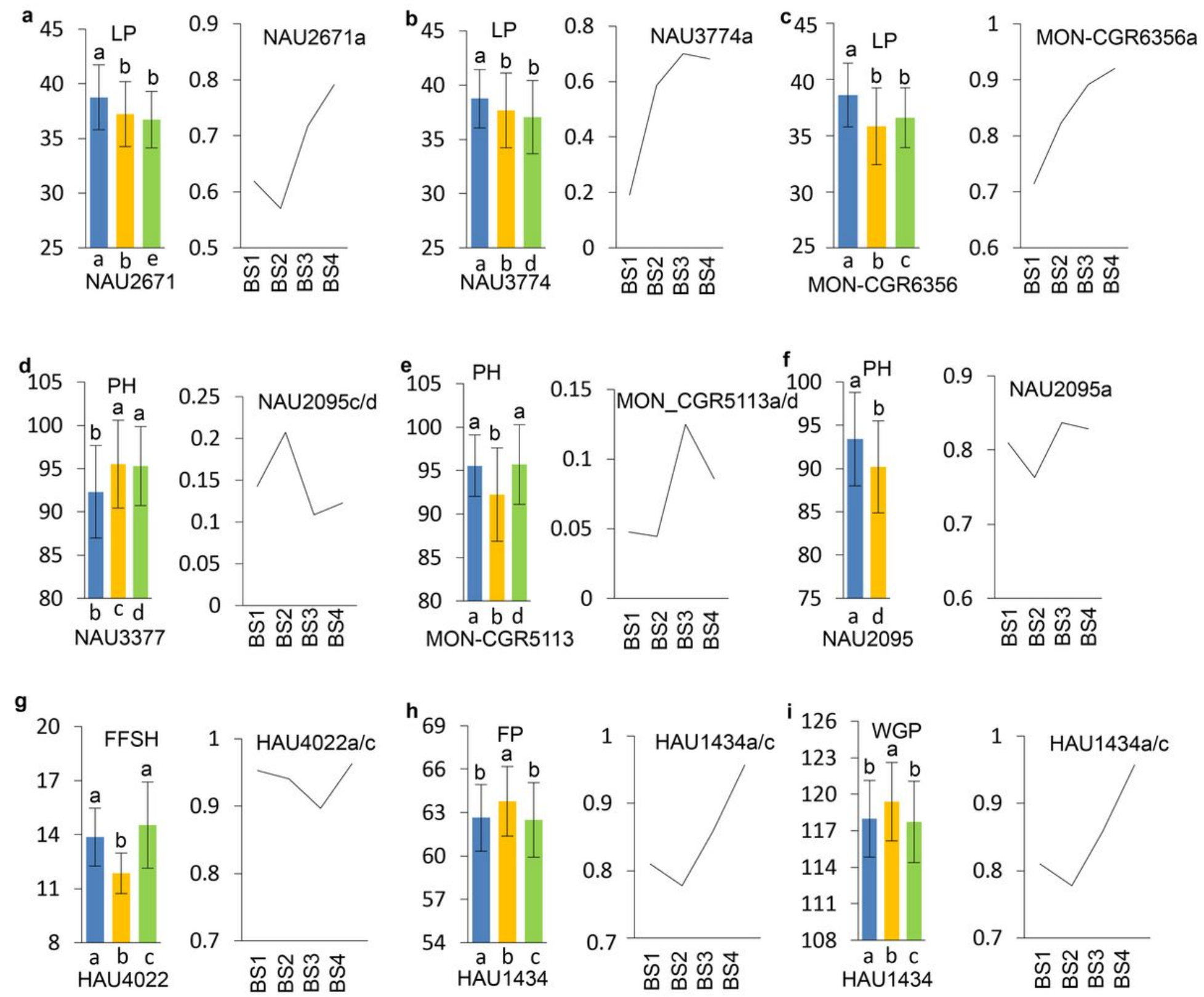

Figure 6

The effects of different alleles of the major QTL $(R 2 \geq 3.0)$ and the favorable allele frequency trends in the four breeding stages. The phenotypic statistics of different alleles of NAU2671, NAU3774, MONCGR6356, NAU3377, MON-CGR5113, NAU2095, HAU4022, and HAU1434 locus was shown in a-i (left chart). And the favorable allele frequency trends in the four breeding stages are shown in a-i (right chart). 

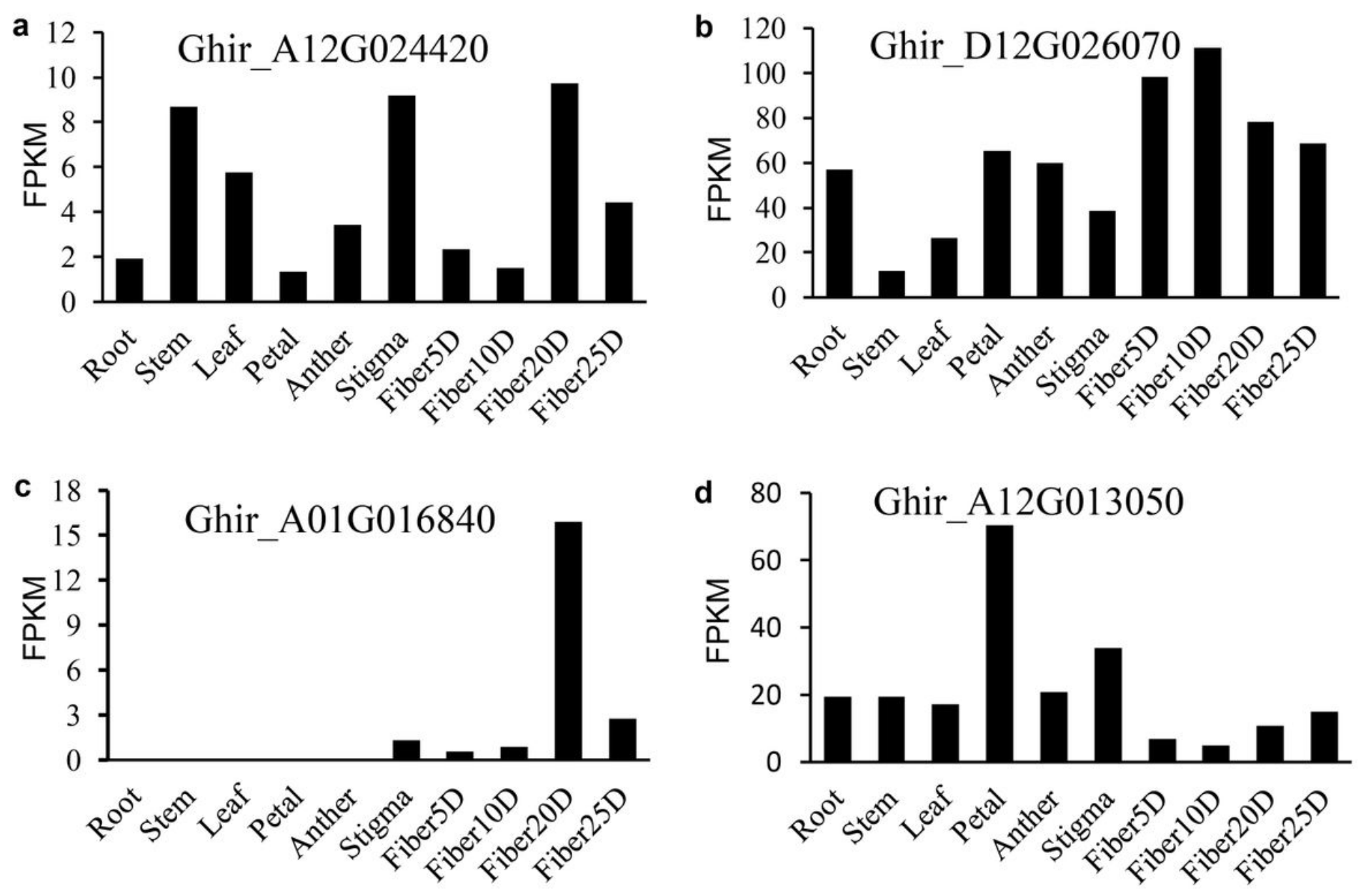

Figure 7

Expression profiles of candidate genes in various tissues. a. Tissue expression of Ghir_A12G024420. b. tissue expression of Ghir_D12G026070. c. tissue expression of Ghir_A01G016840. d. Tissue expression of Ghir_A12G013050.

\section{Supplementary Files}

This is a list of supplementary files associated with this preprint. Click to download.

- Tables1.xls

- TableS2.xIsx

- TableS3.xIsx

- TableS4.xIsx

- TableS5.xIsx

- TableS6.xIsx

- FigureS1.tif 